\title{
Prática clínica de enfermagem paliativa em pediatria
}

\author{
Clinical practice of palliative nursing in pediatrics \\ Práctica clínica de enfermería paliativa en pediatría
}

\section{Resumo}

Os cuidados paliativos são utilizados em pacientes que enfrentam doenças que não possuem cura e comprometam a sua sobrevida. No instante que a doença é descoberta em um paciente infantil, o enfermeiro deve oferecer todo o acompanhamento, suporte psicossocial e espiritual para a criança e seus familiares, com a finalidade de oferecer melhores condições de vida durante todo o percurso do tratamento até processo de partida, que pode levar anos. O objetivo deste estudo foi analisar a prática clínica de enfermagem nos cuidados paliativos realizados em pacientes infantojuvenis. Este estudo é uma pesquisa descritiva, com busca de artigos realizada em bibliotecas digitais. Foi possível constatar neste estudo que os cuidados paliativos evoluíram muito ao longo dos séculos e tornaram-se uma prática profissional, executada não somente pela equipe de enfermagem, mas por toda a equipe multidisciplinar. No século passado os pacientes eram isolados em um leito terminal e esperavam pela morte, sem alívio nenhum de sinais e sintomas. O cenário atual é diferente e exige do profissional uma assistência que leve em conta a dor, possíveis complicações físicas, psicossociais e espirituais do paciente e familiares. O enfermeiro, juntamente com a equipe multidisciplinar, deve apoiar e oferecer suporte emocional, psicológico, espiritual e alívio da dor. Por fim, destaca-se que todo profissional que atua com paciente em fase final de vida deve entender que fez o máximo para suavizar o dor do enfermo e sua família.

Palavras-chave: Cuidados de enfermagem; Cuidados paliativos; Pediatria.

\begin{abstract}
Palliative care is used in patients who face illnesses that have no cure and compromise their survival. The moment the disease is discovered in a child patient, the nurse must offer all the monitoring, psychosocial and spiritual support for the child and their families, to offer better living conditions throughout the course of treatment until the departure process, which can take years. The aim of this study was to analyze the clinical nursing practice in palliative care performed in children and adolescents. This study is descriptive research, with a search for articles carried out in digital libraries. It was possible to observe in this study that palliative care has evolved a lot over the centuries and has become a professional practice, performed not only by the nursing team, but by the entire multidisciplinary team. In the last century, patients were isolated in a terminal bed and waited for death, without any relief of signs and symptoms. The current scenario is different and requires professional assistance that considers the pain, possible physical, psychosocial, and spiritual complications of the patient and family. Nurses, together with the multidisciplinary team, must support and offer emotional, psychological, spiritual and pain relief support. Finally, it is noteworthy that every professional who works with patients in the final stage of life must understand that they did their best to ease the pain of the patient and their family.
\end{abstract}

Keywords: Nursing care, Palliative care; Pediatrics. 


\begin{abstract}
Resumen
Los cuidados paliativos se utilizan en pacientes que enfrentan enfermedades que no tienen cura y comprometen su supervivencia. En el momento en que se descubre la enfermedad en un paciente infantil, la enfermera debe ofrecer todo el seguimiento, apoyo psicosocial y espiritual al niño y a sus familias, con el fin de ofrecer mejores condiciones de vida durante todo el curso del tratamiento hasta el proceso de salida, que puede llevar años. El objetivo de este estudio fue analizar la práctica clínica de enfermería en cuidados paliativos realizada en niños y adolescentes. Este estudio es una investigación descriptiva, con búsqueda de artículos realizada en bibliotecas digitales. En este estudio se pudo observar que los cuidados paliativos han evolucionado mucho a lo largo de los siglos y se han convertido en una práctica profesional, realizada no solo por el equipo de enfermería, sino por todo el equipo multidisciplinario. En el último siglo, los pacientes fueron aislados en un lecho terminal y esperaron la muerte, sin ningún alivio de los signos y síntomas. El escenario actual es diferente y requiere una asistencia profesional que tenga en cuenta el dolor, las posibles complicaciones físicas, psicosociales y espirituales del paciente y su familia. Las enfermeras, junto con el equipo multidisciplinario, deben apoyar y ofrecer apoyo emocional, psicológico, espiritual y de alivio del dolor. Finalmente, es de destacar que todo profesional que trabaja con pacientes en la etapa final de la vida debe comprender que hicieron todo lo posible para aliviar el dolor del paciente y su familia.
\end{abstract}

Palabras clave: Cuidados de enfermería; Cuidados paliativos; Pediatría.

\title{
1. Introdução
}

Os cuidados paliativos são utilizados em pacientes que enfrentam doenças que não possuem cura e comprometam a sua sobrevida. O enfermeiro direciona a assistência de enfermagem para promover a qualidade de vida do paciente e sua família, por meio do suporte emocional, físico, psicossocial e espiritual. O cuidado paliativo apresenta princípios distintos que estão dispostos da seguinte maneira: considerar a morte como um processo natural, não acelerar nem adiar a morte, estimular o paciente a viver ativamente quanto possível até o momento da sua morte, proporcionar alívio da dor, náuseas e vômitos, melhorar a qualidade de vida, influenciar positivamente o curso da doença, oferecer suporte emocional para auxiliar os familiares durante o processo saúde-doença do paciente e no momento de luto (Santos et al., 2017; OMS, 2012).

Conforme Flauzino et al. (2021) quando o tratamento curativo não é mais uma opção, tanto o paciente, como seus familiares passam por uma angústia intensa que está ligada diretamente ao medo da morte. Essa sensação tende a aumentar quando os familiares não recebem informações adequadas sobre o estado de saúde do paciente, o que pode ocasionar uma crise de desespero tanto para o paciente quanto a sua família. Para Medeiros et al. (2020), a essa falta de informação clara e precisa agrava a angústia e ansiedade. Neste momento, os entes mais próximos vão em busca de qualquer tratamento alternativo de cura.

No instante que a doença é descoberta em um paciente infantil, o enfermeiro deve oferecer todo o acompanhamento, suporte psicossocial e espiritual para a criança e seus familiares, com a finalidade de oferecer melhores condições de vida durante todo o percurso do tratamento até processo de partida, que pode levar anos. Nesse período, a equipe de enfermagem vai lidar constantemente com a sensação de fraqueza, vulnerabilidade e angústia dos familiares, pois a perda de uma criança altera o que se considera como sucessão natural, ou seja, em que os pais se vão antes dos filhos (Cesário et al., 2021b). O enfermeiro faz parte da equipe multidisciplinar e acompanha todo o desfecho do caso até a morte do paciente, por isso, o cuidado paliativo deve ser realizado de forma humanizada e integrado com a equipe multiprofissional para esclarecer, informar e ajudar o paciente e familiares neste período difícil (Cesário et al.,2021a).

Além do apoio emocional e psicológicos aos familiares, a assistência de enfermagem deve contribuir para o alívio da dor, diarreia, estomatite, náuseas e vômitos. A enfermagem ajuda o paciente a adaptar-se a mudança de vida que foi imposta pela doença e promove a reflexão necessária para enfrentar a condição de ameaça a sua própria vida (Cesário et al., 2021b).

Conforme Campos, Silva e Silva (2019) o enfermeiro realiza a prática clínica paliativa voltada para integralidade, que envolve as necessidades físicas, emocionais e espirituais do paciente, o que exige a participação efetiva de toda a equipe multiprofissional, para que assim seja possível contribuir para a melhor qualidade de vida do paciente durante todo o tratamento paliativo. Os cuidados paliativos estão relacionados às práticas voltadas para a melhoria da qualidade de vida do paciente e sua 
família durante o enfrentamento de problemas associados à doença, porém muitos profissionais da saúde não são adeptos à prática paliativa por desconhecimento desse tipo de abordagem e por não entenderem a importância dessa abordagem junto ao paciente e família. Ao considerar que o enfermeiro vivencia e executa a assistência de enfermagem sobre cuidados paliativos ao paciente no final da vida, independentemente da idade, levantou-se o seguinte problema de pesquisa: como é a abordagem do enfermeiro nos cuidados paliativos para pacientes infantojuvenis? O objetivo deste estudo foi analisar a prática clínica de enfermagem nos cuidados paliativos realizados em pacientes infantojuvenis.

\section{Metodologia}

Para alcançar o objetivo deste estudo, optou-se pela realização de uma pesquisa descritiva, que de acordo com Cesário, Flauzino e Mejia (2020) é direcionada para responder alguma dúvida ou questionamento e que pode incluir artigos científicos publicados em periódicos acadêmicos. Dessa forma, inicialmente buscou-se reunir evidências para responder ao questionamento de pesquisa. Na etapa inicial, a busca foi realizada em bibliotecas digitais, que permitem uma busca em bases de dados próprias por meio da utilização de descritores padronizados. No DeCS (Descritores em Ciências da Saúde), foram encontrados os seguintes descritores: cuidados de enfermagem, cuidados paliativos e pediatria, que representam as strings de busca.

Os bancos de dados utilizados foram o Google Acadêmico, BVS (biblioteca virtual em saúde) e SciELO (Scientific Electronic Library Online). No Google Acadêmico utilizou-se cada um dos descritores entre aspas (“"). Na BVS foi utilizado a opção pesquisa avançada, selecionada as bases da BDENF (Banco de Dados em Enfermagem), LILACS (Literatura LatinoAmericana e do Caribe em ciências da saúde) e o operador lógico booleano "OR" e "AND". Na SciELO, foi utilizada a opção pesquisa avançada e o operador lógico booleano "OR" e " $A N D$ ".

Foram estabelecidos como critérios de inclusão: artigos acadêmicos publicados entre 2015 e 2021, na língua portuguesa, disponíveis de forma completa nos bancos de dados já mencionados, que respondessem à pergunta de pesquisa. Excluíram-se artigos repetidos encontrados nas bases de dados, resumos, artigos inferiores a 2015, artigos que não respondiam o problema da pesquisa e aqueles que não estavam publicados em revista acadêmica com ISSN (International Standard Serial Number). A coleta dos dados foi realizada no mês de março, por dois pesquisadores de forma independente. Os resultados das buscas pelos dados e do número final de publicações nessa revisão foram apresentados conforme orientações do JBI, na forma de fluxograma PRISMA (Peters, 2015), como mostra a Figura 1: 
Figura 1. Diagrama de fluxo dos artigos encontrados.

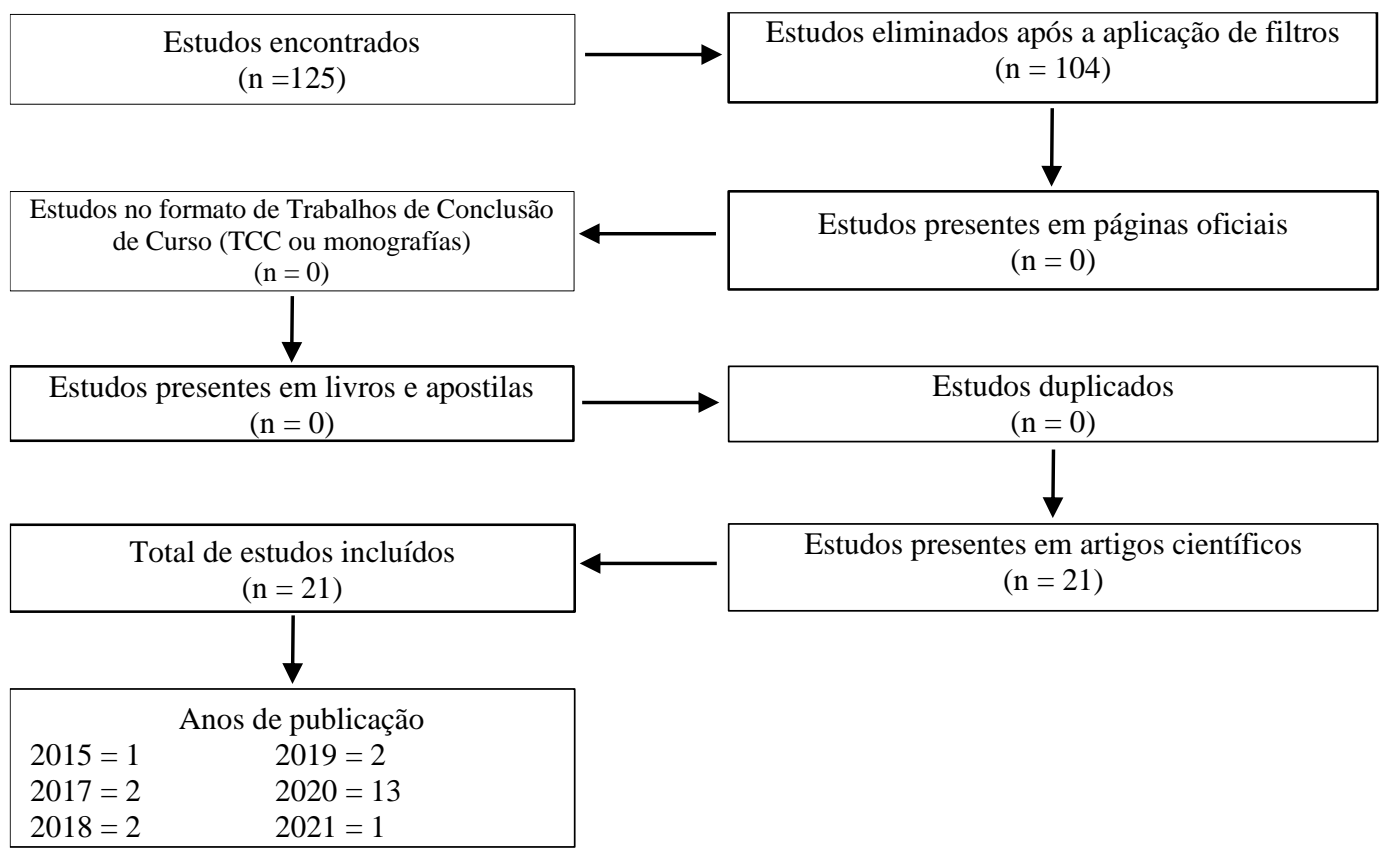

Fonte: Autores (2021).

Inicialmente foram encontrados 125 estudos e após a aplicação dos filtros, conforme os critérios de inclusão e exclusão, foram eliminados 104 estudos. Não foram considerados estudos duplicados, monografias, apostilas e livros. Ao final foram incluídos 21 artigos científicos.

\section{Resultados}

A Tabela 1 foi desenvolvida para mostrar a distribuição inicial dos artigos científicos encontrados nas bases de dados da BVS, SciELO e Google Acadêmico. É possível notar que o maior aproveitamento de periódicos foi nas bases da SciELO e BVS com 8 artigos incluídos de cada base de dados, no Google acadêmico teve somente 5 artigos científicos, o que resultou em uma amostra final de 21 artigos científicos de periódicos diferentes.

Tabela 1. Artigos inclusos por base de dados.

\begin{tabular}{|lllllll|}
\multicolumn{1}{c}{ BVS } & \multicolumn{2}{c}{ ScIELO } & \multicolumn{2}{c|}{ Google Acadêmico } & Amostra final \\
\hline Total & 40 & Total & 30 & Total & 55 & \\
\hline Excluídos & 32 & Excluídos & 22 & Excluídos & 50 & 21 artigos \\
\hline Incluídos & 08 & Incluídos & 08 & Incluídos & 05 & \\
\multicolumn{5}{c}{ Fonte: Autores (2021). }
\end{tabular}

O Quadro 1 foi desenvolvido para apresentar um melhor entendimento dos periódicos selecionados nas bases de dados supracitadas acima. No Quadro 1 é possível notar a relação dos artigos científicos com os objetivos da pesquisa e este foi elaborado com a utilização das seguintes variáveis: autor, título, objetivo central e tipo de estudo. Este quadro contribuiu para realizar a discussão do trabalho, pois a organização dos estudos permitiu uma melhor comparação dos assuntos abordados pelos autores. 
Quadro 1. Artigos incluídos neste estudo.

\begin{tabular}{|c|c|c|c|}
\hline Autor & Título & Objetivo & Tipo de estudo \\
\hline Rolim et al., 2020 & $\begin{array}{l}\text { Teorias científicas de saúde } \\
\text { no cuidado ao paciente } \\
\text { oncológico: }\end{array}$ & $\begin{array}{l}\text { Analisar o uso das teorias de saúde no cuidado } \\
\text { a pacientes oncológicos, destacando os } \\
\text { principais eixos na assistência de enfermagem } \\
\text { oncológica. }\end{array}$ & Revisão integrativa. \\
\hline $\begin{array}{l}\text { Alecrim, Miranda \& } \\
\text { Ribeiro, } 2020\end{array}$ & $\begin{array}{l}\text { Percepção do paciente } \\
\text { oncológico em cuidados } \\
\text { paliativos sobre a família e a } \\
\text { equipe de enfermagem. }\end{array}$ & $\begin{array}{l}\text { Apresentar a percepção do paciente } \\
\text { oncológico em cuidados paliativos quanto à } \\
\text { importância da família e da equipe de } \\
\text { enfermagem durante o tratamento. }\end{array}$ & $\begin{array}{l}\text { Estudo descritivo e } \\
\text { exploratório. }\end{array}$ \\
\hline Silva et al., 2020 & $\begin{array}{lrr}\text { Transição para } & \text { os } & \text { cuidados } \\
\text { paliativos: } & \text { ações } \\
\text { facilitadoras } & \text { para } & \text { uma } \\
\text { comunicação centrada } & \text { no } \\
\text { cliente oncológico. }\end{array}$ & $\begin{array}{l}\text { Analisar como o cliente oncológico avalia a } \\
\text { comunicação na transição para os cuidados } \\
\text { paliativos; identificar suas necessidades e } \\
\text { preferências acerca dessa comunicação } \\
\text { relacionadas ao seu prognóstico, tomada de } \\
\text { decisão e participação familiar. }\end{array}$ & $\begin{array}{l}\text { Pesquisa } \\
\text { convergente. }\end{array}$ \\
\hline $\begin{array}{l}\text { Silva \& Conceição, } \\
2020\end{array}$ & $\begin{array}{l}\text { Cuidados paliativos de } \\
\text { enfermagem a pacientes com } \\
\text { feridas neoplásicas. }\end{array}$ & $\begin{array}{l}\text { Descrever as evidências científicas } \\
\text { disponíveis na literatura sobre os cuidados } \\
\text { paliativos de enfermagem a pacientes com } \\
\text { feridas neoplásicas. }\end{array}$ & Revisão integrativa. \\
\hline Dias et al., 2020 & $\begin{array}{l}\text { Dissertações e teses sobre } \\
\text { cuidados paliativos } \\
\text { oncologia pediátrica: } \\
\text { estudo } \\
\text { bibliométrico. }\end{array}$ & $\begin{array}{l}\text { Analisar indicadores bibliométricos de } \\
\text { estudos oriundos de dissertações e teses sobre } \\
\text { cuidados paliativos em oncologia pediátrica } \\
\text { defendidas em Programas de Pós-Graduação } \\
\text { no cenário brasileiro. }\end{array}$ & Estudo bibliométrico. \\
\hline $\begin{array}{l}\text { Lopes-Júnior et al., } \\
2020\end{array}$ & $\begin{array}{l}\text { Eficácia das } \text { terapias } \\
\text { complementares no manejo } \\
\text { da dor oncológica em } \\
\text { cuidados paliativos: revisão } \\
\text { sistemática. }\end{array}$ & $\begin{array}{l}\text { sintetizar o conhecimento e avaliar } \\
\text { criticamente as evidências provenientes de } \\
\text { ensaios clínicos controlados randomizados } \\
\text { sobre a eficácia das terapias complementares } \\
\text { no manejo da dor oncológica em pacientes } \\
\text { adultos com câncer em cuidados paliativos. }\end{array}$ & Revisão sistemática. \\
\hline $\begin{array}{l}\text { Neves, Muniz \& } \\
\text { Reis. } 2020\end{array}$ & $\begin{array}{lrr}\text { Avaliação de } & \text { sintomas em } \\
\text { pacientes } & & \text { oncológicos } \\
\text { internados } & \text { em } & \text { unidade de } \\
\text { cuidados } & & \text { paliativos } \\
\text { exclusivos } & & \end{array}$ & $\begin{array}{l}\text { Avaliar os sintomas em pacientes oncológicos } \\
\text { internados em uma Unidade de Cuidados } \\
\text { Paliativos exclusivos. }\end{array}$ & Estudo quantitativo. \\
\hline Sousa et al., 2019 & $\begin{array}{l}\text { Instrumento assistencial de } \\
\text { enfermagem em cuidados } \\
\text { paliativos para centro de } \\
\text { terapia intensiva pediátrica } \\
\text { oncológica. }\end{array}$ & $\begin{array}{l}\text { Elaborar um instrumento assistencial de } \\
\text { enfermagem para guiar o atendimento às } \\
\text { crianças e adolescentes com câncer em } \\
\text { cuidados paliativos internados no Centro de } \\
\text { Terapia Intensiva Pediátrica Oncológica. }\end{array}$ & $\begin{array}{l}\text { Pesquisa } \\
\text { metodológica. }\end{array}$ \\
\hline $\begin{array}{l}\text { Guimarães et al., } \\
2017\end{array}$ & $\begin{array}{llr}\text { Cuidado } & \text { paliativo } & \text { em } \\
\text { oncologia } & \text { pediátrica } & \text { na } \\
\text { formação do enfermeiro. } & \end{array}$ & $\begin{array}{l}\text { Identificar e descrever a visão dos } \\
\text { acadêmicos de enfermagem sobre os cuidados } \\
\text { paliativos em oncologia pediátrica durante a } \\
\text { graduação. }\end{array}$ & $\begin{array}{l}\text { Pesquisa } \\
\text { exploratória. }\end{array}$ \\
\hline $\begin{array}{l}\text { Cunha, Pitombeira } \\
\text { \& Panzetti, } 2018\end{array}$ & $\begin{array}{l}\text { Cuidado } \\
\text { oncológico: } \\
\text { cuidadores. }\end{array}$ & $\begin{array}{l}\text { Descrever e analisar a percepção do cuidador } \\
\text { principal frente a um familiar em cuidado } \\
\text { paliativo e traçar o perfil sociodemográfico } \\
\text { dos cuidadores familiares. }\end{array}$ & $\begin{array}{l}\text { Pesquisa exploratória } \\
\text { e descritiva. }\end{array}$ \\
\hline
\end{tabular}




\begin{tabular}{|c|c|c|c|}
\hline Almeida et al., 2020 & $\begin{array}{l}\text { A relação entre o enfermeiro } \\
\text { e o paciente nos cuidados } \\
\text { paliativos oncológicos. }\end{array}$ & $\begin{array}{l}\text { Avaliar a relação e vivência do enfermeiro, } \\
\text { relativos aos cuidados com os pacientes } \\
\text { oncológicos. }\end{array}$ & $\begin{array}{l}\text { Pesquisa exploratório } \\
\text { e qualitativa. }\end{array}$ \\
\hline Souza et al., 2018 & $\begin{array}{l}\text { Cuidados paliativos } \\
\text { pediátricos: análise de } \\
\text { estudos de enfermagem. }\end{array}$ & $\begin{array}{l}\text { Analisar as evidências científicas acerca dos } \\
\text { cuidados paliativos pediátricos. }\end{array}$ & $\begin{array}{l}\text { Estudo } \\
\text { Bibliométrico. }\end{array}$ \\
\hline Lacerda et al., 2020 & $\begin{array}{l}\text { Retirada da ventilação } \\
\text { mecânica } \\
\text { procedimento paliativo emo } \\
\text { uma unidade de terapia } \\
\text { intensiva brasileira. }\end{array}$ & $\begin{array}{l}\text { Descrever as características e os desfechos de } \\
\text { pacientes submetidos à retirada da ventilação } \\
\text { mecânica e comparar a pacientes com } \\
\text { ventilação mecânica e limitações de terapias } \\
\text { de suporte à vida. }\end{array}$ & Estudo retrospectivo. \\
\hline Pinho et al., 2020 & $\begin{array}{l}\text { Repercussões dos cuidados } \\
\text { paliativos pediátricos: } \\
\text { revisão integrativa. }\end{array}$ & $\begin{array}{l}\text { Efeitos dos serviços de cuidados paliativos } \\
\text { pediátricos sobre a instituição hospitalar e a } \\
\text { qualidade de vida de pacientes e seus } \\
\text { familiares. }\end{array}$ & Estudo qualitativo. \\
\hline $\begin{array}{l}\text { Lourenção } \quad \& \\
\text { Troster } 2020\end{array}$ & $\begin{array}{l}\text { Fim de vida em unidades de } \\
\text { terapia intensiva pediátrica. }\end{array}$ & $\begin{array}{l}\text { Analisa as condutas de fim de vida em } \\
\text { unidades de terapia intensiva pediátrica } \\
\text { brasileiras. }\end{array}$ & $\begin{array}{l}\text { Estudo } \\
\text { observacional, } \\
\text { retrospectivo, } \\
\text { multicêntrico. }\end{array}$ \\
\hline Lima et al., 2020 & $\begin{array}{l}\begin{array}{l}\text { Dinâmica da oferta de } \\
\text { cuidados }\end{array} \\
\text { pediátricos: estudo de casos } \\
\text { múltiplos. }\end{array}$ & $\begin{array}{l}\text { Analisar a dinâmica que envolve a oferta de } \\
\text { cuidados paliativos para crianças elegíveis, na } \\
\text { perspectiva de profissionais e familiares. }\end{array}$ & $\begin{array}{l}\text { Estudo exploratório } \\
\text { qualitativo. }\end{array}$ \\
\hline $\begin{array}{l}\text { Medeiros et al., } \\
2020\end{array}$ & $\begin{array}{l}\text { Conflitos bioéticos nos } \\
\text { cuidados de fim de vida. }\end{array}$ & $\begin{array}{l}\text { Identificar os principais conflitos entre } \\
\text { paciente em cuidados de fim de vida, } \\
\text { familiares e equipe de saúde sob a ótica da } \\
\text { ética principia lista. }\end{array}$ & Revisão integrativa \\
\hline $\begin{array}{l}\text { Campos, Silva, \& } \\
\text { Silva. } 2019\end{array}$ & $\begin{array}{l}\text { Comunicação em cuidados } \\
\text { paliativos: equipe, paciente e } \\
\text { família. }\end{array}$ & $\begin{array}{l}\text { Avaliar a comunicação na assistência } \\
\text { paliativa e sua influência na relação entre } \\
\text { equipe, paciente e família. }\end{array}$ & $\begin{array}{l}\text { Pesquisa qualitativa, } \\
\text { descritiva e } \\
\text { exploratória. }\end{array}$ \\
\hline Silva et al., 2015 & $\begin{array}{l}\text { Cuidados paliativos na } \\
\text { assistência de alta } \\
\text { complexidade em oncologia: } \\
\text { percepção de enfermeiros. }\end{array}$ & $\begin{array}{l}\text { Identificar as dificuldades enfrentadas na } \\
\text { prestação da assistência à pessoa } \\
\text { hospitalizada no contexto dos cuidados } \\
\text { paliativos em um Centro de Assistência de } \\
\text { Alta Complexidade em Oncologia do estado } \\
\text { do Rio de Janeiro, na percepção dos } \\
\text { enfermeiros. }\end{array}$ & $\begin{array}{l}\text { Pesquisa } \\
\text { descritiva, } \\
\text { abordagem } \\
\text { qualitativa. }\end{array}$ \\
\hline Santos et al., 2017 & $\begin{array}{l}\text { Planejamento da assistência } \\
\text { ao paciente em cuidados } \\
\text { paliativos na terapia } \\
\text { intensiva oncológica. }\end{array}$ & $\begin{array}{l}\text { Analisar o entendimento dos profissionais de } \\
\text { saúde acerca da assistência ao paciente em } \\
\text { cuidados ao fim da vida na unidade de terapia } \\
\text { intensiva (UTI) oncológica, e discutir os } \\
\text { objetivos que buscam alcançar ao planejar a } \\
\text { assistência na perspectiva dos cuidados } \\
\text { paliativos. }\end{array}$ & $\begin{array}{l}\text { Estudo descritivo, } \\
\text { com abordagem } \\
\text { qualitativa. }\end{array}$ \\
\hline $\begin{array}{l}\text { Silva, Assis } \quad \& \\
\text { Pinto, } 2021\end{array}$ & $\begin{array}{l}\text { Cuidados Paliativos na } \\
\text { Criança com Câncer: o papel } \\
\text { do enfermeiro na assistência } \\
\text { do cuidar }\end{array}$ & $\begin{array}{l}\text { Realizar uma revisão da literatura acerca da } \\
\text { atuação do enfermeiro na atenção aos } \\
\text { cuidados paliativos na criança com câncer. }\end{array}$ & Revisão de literatura. \\
\hline
\end{tabular}

Fonte: Autores (2021). 


\section{Discussão}

Os primeiros relatos históricos relacionados ao cuidado paliativo ocorreram durante a Idade Média, período em que a assistência aos desfalecidos, doentes, órfãos e leprosos, eram realizados para o acolhimento, proteção e alívio do sofrimento, concedido por pessoas comuns que ofereciam caridades aos necessitados. Como muitas vezes não havia cura para a doença, as pessoas eram acolhidas para que tivessem seu sofrimento amenizado. No século XVII, a Igreja Católica iniciou os cuidados aos exilados, portadores de doenças graves com auxílio da comunidade, formada por pessoas comuns. Apenas na década 1900, foram iniciados os cuidados em "hospices", que eram lugares destinados a pessoas portadoras de doenças letais. No século XX, os cuidados paliativos passaram a ser realizados de maneira institucionalizada, o que deu origem a uma nova forma de assistência aos doentes fora de capacidade terapêuticas curativas (Lima et al., 2020).

Antigamente a prática clínica era muito diferente, principalmente em relação aos cuidados paliativos, que praticamente inexistiam. Muitas vezes as crianças eram colocadas juntos com enfermos adultos. Os pacientes eram isolados em um leito terminal e esperavam pela morte, sem alívio nenhum de sinais e sintomas. Com o passar dos anos e com a implementação da humanização os cuidados paliativos evoluíram e hoje é uma prática realizada em diversos hospitais no Brasil (Campos, Silva, \& Silva. 2019).

Cicely Saunders, pioneira nos cuidados paliativos, criou em 1967 a instituição conhecida como St. Christopher's Hospices, na cidade de Londres, capital da Inglaterra. Este nosocômio era responsável por acolher doentes em estágio terminal, proporcionar o conforto da angústia, controlar sinais e sintomas e oferecer melhor qualidade de vida (Medeiros et al., 2020).

Conforme descrito por Cunha, Pitombeira e Panzetti, (2018), a prática de cuidados paliativos adquiriu ao longo do tempo um significado amplo para agregar à concepção de cuidado integral, ativo e continuado, voltado ao paciente e a sua família. Para que o cuidado paliativo seja realizado de modo satisfatório, é indispensável a formação de uma aliança entre a equipe multidisciplinar, que é composta por enfermeiro, médico, assistente social, psicóloga, terapeuta ocupacional, fisioterapeuta e fonoaudiólogo (Silva, Assis \& Pinto, 2021

Neves, Muniz e Reis. (2020) descreve como o enfermeiro é o responsável por desenvolver a prática clínica paliativa com uma percepção humanística, que deve entender as limitações do paciente e criar um vínculo de confiança com a finalidade de propiciar a melhor qualidade de vida para o paciente durante o enfrentamento da doença. $\mathrm{O}$ enfermeiro durante a implementação dos cuidados deve levar em conta a dor, complicações físicas, psicossociais e espirituais do paciente e familiares (Santos et al., 2017).

Para Lopes-Júnior et al. (2020), durante o atendimento pediátrico, os profissionais da enfermagem devem considerar que são envolvidos, pelo seu paciente, em um mundo de desenhos, cores, fantasias, desejos, manias, em que a brincadeira é uma necessidade diária da criança, que não deve ser alterado pela hospitalização. Por participar desse planeta mágico, os profissionais de enfermagem acabam por multiplicar a sua rede de vínculos afetivos, o que pode não se restringir apenas a uma atuação profissional e partir para um lado emocional que envolve familiares, que pode gerar desequilíbrio emocional de ambas as partes (Rolim et al., 2020).

Essa dificuldade no manejo de crianças em fase final de vida é ainda mais acentuada aos profissionais técnicos de enfermagem, devido ao vínculo afetivo criado com o paciente. Para o enfermeiro opor-se a este problema deverá educar a equipe de por meio de ensino e treinamento fundamentado nos princípios da ética profissional, com objetivo de oferecer a assistência digna ao paciente nos seus últimos dias de vida. (Alecrim, Miranda \& Ribeiro, 2020).

A construção de um vínculo afetivo no paciente em fase final de vida é um trabalho árduo, pois o profissional se depara com o desprazer de familiares, que ao receberem a notícia que o seu ente querido possui uma doença em que não há a possibilidade de cura, o que leva a um ambiente, que antes era de esperança, a transformar-se em local de sofrimento e dor. Para 
amenizar a situação é importante o já citado apoio psicológico, assim como o esclarecimento das dúvidas da família sobre os cuidados paliativos (Silva et al., 2015). A proteção de danos ao indivíduo, ainda que não apresente capacidade de cura, é uma das tarefas mais importantes do enfermeiro, que deve direcionar sua prática clínica para proporcionar uma vida mais confortável durante o tratamento e quando chegar o momento da partida, que ela seja digna e sem dor.

De acordo com Guimarães et al. (2017), os pais, no momento que recebem a notícia que o melhor tipo de cuidado para o filho ou filha são os cuidados paliativos, entram em um estado de negação e procuram pela possibilidade da cura milagrosa. Os familiares sofrem ainda mais quando não encontram o tratamento sonhado e isso pode contribuir para que na mente da criança, seja criado um mundo fantasioso com pré-conceitos e medos sobre a morte. Para que isto não aconteça o enfermeiro deve trabalhar em conjunto com a equipe multiprofissional para oferecer as informações mais precisas possíveis para a criança e familiares (Almeida et al., 2020). Em cada faixa etária a criança irá absorver a informação de uma maneira diferente e cabe ao enfermeiro adaptar sua linguagem de forma que o paciente consiga entender a mensagem que o profissional precisa transmitir.

Para Sousa et al. (2019) as crianças com doenças agudas ou crônicas que estão em risco de morte apresentam elevado quadro depressivo, que progride de forma acelerada e está ligado a fatores como a idade, dor, efeitos colaterais de medicamentos e atitudes negativas de acompanhantes. A depressão é um problema difícil de se superar e a equipe deve atuar em conjunto para oferecer um atendimento que garanta o bem-estar físico e mental, que melhore de fato a qualidade de vida e contribua para amenizar o sofrimento do paciente infantojuvenil durante este processo doloroso (Souza et al., 2018).

Dias et al. (2020) descreve que as crianças que estão em cuidados paliativos sofrem bastante com a dor e isso interfere diretamente no bem-estar do paciente, o que deixa os familiares estressados com a situação, pois já estão com o estado psicológico e emocional abalados. Para realizar o controle álgico o enfermeiro deve avaliar o paciente por meio de escalas ou instrumentos padronizados para mensurar a dor e oferecer e ajustar o analgésico prescrito pelo médico, além de tranquilizar o paciente e ensinar medidas não medicamentosas para o alívio da dor (Lacerda et al., 2020).

Um aspecto complicado e doloroso para o enfermeiro, durante a prática clínica paliativa pediátrica, é suportar a morte da criança (Pinho et al., 2020), pois trata-se de uma experiência complexa, mais difícil de lidar do que a partida de um adulto, pois não faz parte do curso natural da vida. O profissional que atua com pacientes terminais deve entender que fez o máximo para suavizar o dor do enfermo e sua família e contribuiu para o paciente tivesse uma morte digna e honrosa, com o menor sofrimento possível.

\section{Conclusão}

Foi possível constatar neste estudo que os cuidados paliativos evoluíram muito ao longo dos séculos e tornaram-se uma prática profissional, executada não somente pela equipe de enfermagem, mas por toda a equipe multidisciplinar. No século passado os pacientes eram isolados em um leito terminal e esperavam pela morte, sem alívio nenhum de sinais e sintomas. O cenário atual é diferente e exige do profissional uma assistência que leve em conta a dor, possíveis complicações físicas, psicossociais e espirituais do paciente e familiares.

A prática clínica paliativa é a medida de suporte e conforto para aliviar o sofrimento do paciente com objetivo de oferecer uma morte digna e honrosa. O enfermeiro, juntamente com a equipe multidisciplinar, deve apoiar e oferecer suporte emocional, psicológico, espiritual e alívio da dor. Deve ser garantido ao paciente infantojuvenil, o alívio da dor, conforto e qualidade de vida. A família também precisa de amparo, pois sofrem durante a fase terminal da criança e não forem bem orientados e acolhidos, podem repassar a criança o estresse, o que pode ocasionar piora no quadro do paciente.

Muitas vezes a equipe de enfermagem cria vínculos com os enfermos e seus familiares, pois estes pacientes ficam por longos períodos de internação e sensibilizam os profissionais que atendem o paciente. $\mathrm{O}$ enfermeiro responsável pela equipe 
Research, Society and Development, v. 10, n. 12, e57101218102, 2021

(CC BY 4.0) | ISSN 2525-3409 | DOI: http://dx.doi.org/10.33448/rsd-v10i12.18102

deve preparar adequadamente o seu pessoal para lidar com essas situações. Todo profissional que atua com pacientes terminais deve entender que fez o máximo para suavizar o dor do enfermo e sua família.

Apesar da importância do assunto abordado neste estudo, além de ser uma prática antiga, os estudos sobre cuidados paliativos no idioma português ainda são escassos. Dessa forma, os autores esperam que este estudo contribua para estimular novas pesquisas relacionadas aos cuidados paliativos em pediatria.

\section{Referências}

Alecrim, T. D. P., Miranda, J. A. M. \& Ribeiro, B. M. S. S. (2020). Percepção do paciente oncológico em cuidados paliativos sobre a família e a equipe de enfermagem. Revista de Enfermagem do Centro Hospitalar de Setúbal. 14(2):206-212. https://doi.org/10.25242/886882720181479.

Almeida, P. F. Barbosa, M. G. A., Santos, S. M., Silva, E. I. \& Lins, S. R. O. (2020). A relação entre o enfermeiro e o paciente nos cuidados paliativos oncológicos. Brazilian Journal of health Review. 3(2),1465-1483. https://doi.org/10.34119/bjhrv3n2-011

Campos, V. F., Silva, J. M. \& Silva, J. J. (2019). Comunicação em cuidados paliativos: equipe, paciente e família. Revista Bioética. 27(4), 711-8. https://doi.org/ $10.1590 / 1983-80422019274354$

Cesário, J. M. S., Flauzino, V. H. P., Hernandes, L. O., Gomes, D. M. \& Vitorino, P. G. S. (2021a). Assistência de enfermagem ao paciente oncológico submetido à anestesia. Research, Society and Development, 10(5), e31310514798. https://doi.org/10.33448/rsd-v10i5.14798.

Cesário, J. M. S., Flauzino, V. H. P., Hernandes, L. O., Gomes, D. M. \& Vitorino, P. G. S. (2021b). Assistência de enfermagem aos pacientes com cardiotoxicidade induzidas por quimioterápicos. Research, Society and Development. 10(6), e34210615355. https://doi.org/10.33448/rsd-v10i6.15355

Cesário, J. M. S.; Flauzino, V. H. P \& Mejia, J. V. C. (2020) Metodologia científica: Principais tipos de pesquisas e suas caraterísticas. Revista Científica Multidisciplinar Núcleo do Conhecimento. 5(11). 23-33. https://doi.org/10.32749/nucleodoconhecimento.com.br/educacao/tipos-de-pesquisas.

Cunha, A. S., Pitombeira, J. S. \& Panzetti, T. M. N (2018). Cuidado paliativo oncológico: percepção dos cuidadores. Journal of Health \& Biological Sciences. 6(4), 383-390. https://doi.org/10.12662/2317-3076jhbs.v6i4.2191.p383-390.2018.

Dias, K. C. C. O., Batista, P. S. S., Fernandes, M. A., Zaccara, A. A. L., Oliveira, T. C., Vasconcelos, M. F., Oliveira, A. M. M. \& Andrade, F. F. (2020). Dissertações e teses sobre cuidados paliativos em oncologia pediátrica: Estudo bibliométrico. Acta Paulista de Enfermagem. 1(33), 1-8. https://doi.org/10.37689/actaape/2020AO02642.

Flauzino, V. H. P., Vitorino, P. G. S., Hernandes, L. O., Gomes, D. M. \& Cesário, J. M. S. (2021). Paciente cardiopata em tratamento de câncer: Implicações para o cuidado de enfermagem. Research, Society and Development, 10(6), e34310615357. https://doi.org/10.33448/rsd-v10i6.15357

Guimarães, T. M. Silva, L. F., Santo, F. H. E., Moraes, J. R. M. M. \& Pacheco, S. T. A. (2017). Cuidado paliativo em oncologia pediátrica na formação do enfermeiro. Revista Gaúcha Enfermagem. 38(1), e65409. https://doi.org/10.1590/1983-1447.2017.01.65409

Lopes-Júnior, L. C., Rosa, G. S., Pessanha, R. M., Schuab, S. I. P. C., Nunes, K. Z. \& Amorim, M. H. C. (2020). Eficácia das terapias complementares no manejo da dor oncológica em cuidados paliativos: revisão sistemática. Revista Latino-Americana Enfermagem. 1(28), e3377. https://doi.org/10.1590/15188345.4213 .3377

Lacerda, F. H. Checoli, P. G., Silva, C. M. D., Brandão, C. E., Forte, D. N. \& Besen. B. A. M. P. (2020). Retirada da ventilação mecânica como procedimento paliativo em uma unidade de terapia intensiva brasileira. Revista Brasileira Terapia Intensiva. 32(4), 528-534. https://doi.org/10.5935/0103-507X.20200090

Lima, S. F. Lamy, Z. C., Motta, V. B. R., Roma, T. M., Gomes, C. M. R. P. \& Souza, T. P. (2020). Dinâmica da oferta de cuidados paliativos pediátricos: estudo de casos múltiplos. Caderno de Saúde Pública. 36(9), e00164319. https://doi.org/10.1590/0102-311X00164319.

Lourenção, M. L. \& Troster, E. J. (2020). Fim de vida em unidades de terapia intensiva pediátrica. Revista Bioética. 28 (3), 537-42. https://doi.org/10.1590/198380422020283418

Medeiros, M. O. S. F., Meira, M. V., Fraga, F. M. R., Sobrinho, C. L. N., Rosa, D. O. S. \& Rudval, R. S. (2020). Conflitos bioéticos nos cuidados de fim de vida. Revista Bioética. 28(1), 128-34. https://doi.org/10.1590/1983-80422020281375

Neves, K. E. S., Muniz, T. S. \& Reis, K. M. C. (2020). Avaliação de sintomas em pacientes oncológicos internados em unidade de cuidados paliativos exclusivos. Cogitare Enfermagem. 1(25), e71660. https://doi.org/10.5380/ce.v25i0.71660

OMS, Organização Mundial de saúde (2012). National cancer control programmes: policies and managerial guidelines.

Pinho, A. A. A., Nascimento, I. R. C., Ramos, I. W.S. \& Alencar, V. O. (2020). Repercussões dos cuidados paliativos pediátricos: revisão integrativa. Revista Bioética. 28 (4), 710-7. https://doi.org/10.1590/1983-80422020284435

Rolim, I. L. T. P., Pascoal, L. M., Ferreira, A. G. N., Lima, J. F. B., Lisbôa, L. 1. C. \& Costa, T. S. (2020). Teorias científicas de saúde no cuidado ao paciente oncológico: revisão integrativa. Revista de Enfermagem do Centro-Oeste Mineiro. 10(1), e3654. https://doi.org/10.19175/recom.v10i0.3654.

Silva, G. F., Assis, M. T. B. \& Pinto, N. B. F. (2021). Cuidados Paliativos na Criança com Câncer: o papel do enfermeiro na assistência do cuidar. Brazilian Journal of Development. 7(5), 53524-53540. https://doi.org/10.34117/bjdv7n5-655. 
Research, Society and Development, v. 10, n. 12, e57101218102, 2021

(CC BY 4.0) | ISSN 2525-3409 | DOI: http://dx.doi.org/10.33448/rsd-v10i12.18102

Silva, M. M., Moreira, M. C., Zepeda, K. G. M. \& Gaspar, R. B. (2017). Planejamento da assistência ao paciente em cuidados paliativos na terapia intensiva oncológica. Acta Paulista de Enfermagem. 30(3), 295-300. https://doi.org/10.1590/1982-0194201700045.

Silva, E. V. S. \& Conceição, H. N. (2020). Cuidados paliativos de enfermagem a pacientes com feridas neoplásicas. Revista Espaço para a Saúde. Jul.;21(1), 82-94. https://doi.org/10.22421/15177130-2020v21n1p 82

Silva, J. L. R., Cardozo, I. R. C., Souza, S. R. S., Alcântara, L. F. F. L., Silva, C. M. C., Santo, F. H. E. S., Chagas, M. C \& Pinto, A. C. S. (2020). Transição para os cuidados paliativos: ações facilitadoras para uma comunicação centrada no cliente oncológico. Revista Mineira de Enfermagem. 1(24), e1333. https://doi.org/10.5935/1415.2762.20200070

Silva, M. M., Santanda, N. G. M., Santos, M. C., Cirilo, J. D., Barrocas, D. L. R. \& Moreira, M. C. (2015). Cuidados paliativos na assistência de alta complexidade em oncologia: percepção de enfermeiros. Escola Anna Nery Revista de Enfermagem. 19(3), 460-466. https://doi.org/ 10.5935/1414-8145.20150061

Sousa, A. D. R. S., Silva, L. F., Cavalcanti, A. C. D., Góes, F. G. B. \& Moraes, J. R. M. M. (2019). Instrumento assistencial de enfermagem em cuidados paliativos para centro de terapia intensiva pediátrica oncológica. Enfermagem Foco. 10(7), 28-34.

Souza, T. C. F., Correa Júnior, A. J. S, Santana, M. E. \& Carvalho, J. N. (2018). Cuidados paliativos pediátricos: análise de estudos de enfermagem. Revista enfermagem Universidade Federal de Pernambuco. 12(5):1409-22. https://doi.org/10.5205/1981-8963-v12i5a231304p1409-1422-2018 